\title{
Superradiantly stable non-extremal Reissner-Nordström black holes
}

\author{
Jia-Hui Huang ${ }^{1, a}$, Zhan-Feng Mai ${ }^{2}$ \\ ${ }^{1}$ Laboratory of Quantum Engineering and Quantum Materials, School of Physics and Telecommunication Engineering, South China Normal \\ University, Guangzhou 510006, China \\ 2 Department of Physics, Center for Advanced Quantum Studies, Beijing Normal University, Beijing 100875, China
}

Received: 5 March 2015 / Accepted: 25 May 2016 / Published online: 7 June 2016

(C) The Author(s) 2016. This article is published with open access at Springerlink.com

\begin{abstract}
The superradiant stability is investigated for non-extremal Reissner-Nordström black holes. We use an algebraic method to demonstrate that all non-extremal Reissner-Nordström black holes are superradiantly stable against a charged massive scalar perturbation. This improves the results obtained before for non-extremal ReissnerNordström black holes.
\end{abstract}

The stability problem of black hole is an important topic in black hole physics. Regge and Wheeler [1] proved that the spherically symmetric Schwarzschild black hole is stable under perturbations. The stability problems of rotating or charged black holes are complicated due to the significant effect of superradiance. The superradiance effect can occur in both classical and quantum scattering processes [25]. When a charged bosonic wave is impinging on a charged rotating black hole, the wave reflected by the event horizon will be amplified if the wave frequency $\omega$ lies in the following superradiant regime:

$0<\omega<m \Omega+e \Phi$

where $m$ and $e$ are the azimuthal harmonic number and charge of the incoming charged wave, $\Omega$ is the angular velocity of black hole horizon, and $\Phi=Q / r_{H}$ is the electric potential of the black hole [6-12]. This means that when the incoming wave is scattered, the wave extracts rotational energy from the rotating black hole and electric energy from charged black hole. According to the black hole bomb mechanism proposed by Press and Teukolsky [13], if there is a mirror between the black hole horizon and spatial infinity, the amplified wave can be reflected back and forth between the mirror and the black

After this paper was completed, Ref. [31] appeared which addresses the same issue with a different method and gets the same conclusion.

a e-mail: huangjh@m.scnu.edu.cn hole and grows exponentially. This leads to the superradiant instability of the black hole.

The superradiant mechanism has been studied by many authors for the (in)stability problem of black holes [1426]. Recently, for a Kerr black hole under massive scalar perturbation, Hod has proposed a stronger stability regime than before [27]. The extremal and non-extremal charged Reissner-Nordström ( $\mathrm{RN}$ ) black holes have been proved to be stable against a charged massive perturbation [28,29]. Similarly, the analog of the charged RN black hole in string theory has also been proved to be stable under a charged massive scalar perturbation [30].

In fact, up to now, the non-extremal charged RN black hole is proved to be superradiantly stable when the mass $M$ and charge $Q$ of the black hole satisfy $(Q / M)^{2} \leq 8 / 9$ [28,29]. In this paper, we demonstrate that the all non-extremal charged RN black hole is stable against a massive charged scalar perturbation. We find that there is no trapping well outside the black hole, which is separated from the horizon by a potential barrier. As a result, there is no bound state in the superradiant regime, that can lead to the instability of the charged RN black hole.

The metric of the RN black hole (in natural unit $G=c=$ $\hbar=1)$ is

$$
\begin{aligned}
\mathrm{d} s^{2}= & -\left(1-\frac{2 M}{r}+\frac{Q^{2}}{r^{2}}\right) \mathrm{d} t^{2}+\frac{1}{\left(1-\frac{2 M}{r}+\frac{Q^{2}}{r^{2}}\right)} \mathrm{d} r^{2} \\
& +r^{2}\left(\mathrm{~d} \theta^{2}+\sin ^{2} \theta \mathrm{d} \phi^{2}\right),
\end{aligned}
$$

where $M$ and $Q$ are the mass and electric charge of the black hole. The dynamics of a charged massive scalar field perturbation $\Psi$ is governed by the Klein-Gordon equation,

$\left[\left(\nabla^{v}-i q A^{v}\right)\left(\nabla_{v}-i q A_{v}\right)-\mu^{2}\right] \Psi=0$ 
where $q$ and $\mu$ are the charge and the mass of the scalar field. $A_{v}=-\delta_{v}^{0} Q / r$ is the vector potential that describes the spherically symmetric electric field. The solution of the above equation with definite spherical harmonic eigenvalues can be written as

$\Psi_{l m}(t, r, \theta, \phi)=R_{l m}(r) Y_{l m}(\theta, \phi) e^{-i \omega t}$,

where $Y_{l m}$ is the spherical harmonic function, $l$ is the spherical harmonic index, $m$ is the azimuthal harmonic index with $-l \leqslant m \leqslant l$, and $\omega$ is the energy of the mode. The radial Klein-Gordon equation obeyed by $R_{l m}$ (we denote $R_{l m}$ by $R$ in the following) is given by

$\Delta \frac{\mathrm{d}}{\mathrm{d} r}\left(\Delta \frac{\mathrm{d} R}{\mathrm{~d} r}\right)+U R=0$

where $\Delta=r^{2}-2 M r+Q^{2}$ and

$U=\left(\omega r^{2}-q Q r\right)^{2}-\Delta\left[\mu^{2} r^{2}+l(l+1)\right]$.

The inner and outer horizons of the black hole are

$r_{ \pm}=M \pm \sqrt{M^{2}-Q^{2}}$

and it is obvious that

$r_{+}+r_{-}=2 M, \quad r_{+} r_{-}=Q^{2}$.

In order to study the superradiance stability of the black hole against the massive charged perturbation, the asymptotic solutions of the radial wave equation near the horizon and at infinity will be considered with proper boundary conditions. We proceed by defining the tortoise coordinate $y$ by the equation $\frac{\mathrm{d} y}{\mathrm{~d} r}=\frac{r^{2}}{\Delta}$ and a new radial function $\tilde{R}=r R$. The radial wave Eq. (5) can be written as

$\frac{\mathrm{d}^{2} \tilde{R}}{\mathrm{~d} y^{2}}+\tilde{U} \tilde{R}=0$,

where

$\tilde{U}=\frac{U}{r^{4}}-\frac{\Delta}{r^{3}} \frac{\mathrm{d}}{\mathrm{d} r}\left(\frac{\Delta}{r^{2}}\right)$.

It is easy to obtain the asymptotic behavior of the new potential $\tilde{U}$ as

$\lim _{r \rightarrow r_{+}} \tilde{U}=\frac{\left(\omega r_{+}-q Q\right)^{2}}{r_{+}^{2}}, \quad \lim _{r \rightarrow \infty} \tilde{U}=\omega^{2}-\mu^{2}$.

The chosen boundary conditions are ingoing waves at the horizon $(y \rightarrow-\infty)$ and bound states (exponentially decaying modes) at spatial infinity $(y \rightarrow+\infty)$. Then the radial wave equation has the following asymptotic solutions:

$\tilde{R} \sim \begin{cases}e^{-i\left(\omega-\frac{q Q}{r_{+}}\right) y}, & y \rightarrow-\infty\left(r \rightarrow r_{+}\right) \\ e^{-\sqrt{\mu^{2}-\omega^{2}} y}, & y \rightarrow+\infty(r \rightarrow+\infty) .\end{cases}$

It is obvious that when

$\omega^{2}<\mu^{2}$

there is a bound state of the scalar field.

In the following, we prove that there is no trapping well outside the black hole horizon when the parameters of the scalar field and the black hole satisfy the bound state condition (12) and the superradiance condition of the RN black hole,

$0<\omega<q \Phi_{H}=q Q / r_{+}$.

We define a new radial function $\phi$ by $\phi=\Delta^{\frac{1}{2}} R$, then the radial Eq. (5) can be rewritten as

$\frac{\mathrm{d}^{2} \phi}{\mathrm{d} r^{2}}+\left(\omega^{2}-V\right) \phi=0$,

where

$V=\omega^{2}-\frac{U+M^{2}-Q^{2}}{\Delta^{2}}$.

In order to see if there exists a trapping potential outside the horizon, we should analyze the shape of the effective potential $V$. From the following asymptotic behavior of the potential $V$ :

$$
\begin{aligned}
V(r \rightarrow+\infty) \rightarrow & \mu^{2}+\frac{2 M \mu^{2}+2 Q q \omega-4 M \omega^{2}}{r} \\
& +o\left(\frac{1}{r^{2}}\right), \\
V\left(r \rightarrow r_{+}\right) \rightarrow & -\infty,
\end{aligned}
$$

we know there is at least one maximum for $V$ outside the event horizon. It is easy to see that the asymptotic behavior of the derivative of $V$ is

$V^{\prime}=-\frac{2 M \mu^{2}+2 Q q \omega-4 M \omega^{2}}{r^{2}}+o\left(\frac{1}{r^{3}}\right)$.

We can prove the coefficient $2 M \mu^{2}+2 Q q \omega-4 M \omega^{2}>$ 0 when $\omega$ satisfies the superradiance and the bound state conditions. Define a quadratic function $f$ for $\omega$

$$
f(\omega)=-4 M \omega^{2}+2 Q q \omega+2 M \mu^{2} .
$$


It is obvious that there are two zero points for $f$ with opposite sign, and the positive one is

$\omega_{+}=\frac{Q q+\sqrt{Q^{2} q^{2}+8 M^{2} \mu^{2}}}{4 M}$.

To verify $f(\omega)>0$ when $\omega$ satisfies the superradiance and the bound state conditions, we just need to prove $\omega<\omega_{+}$.

Case I: $\omega<\mu \leq q Q / r_{+}$

With the obvious relation $r_{+}>M$, we can get

$$
\omega_{+}=\frac{q Q}{4 M}+\sqrt{\frac{q^{2} Q^{2}}{16 M^{2}}+\frac{\mu^{2}}{2}}>\frac{\mu r_{+}}{4 r_{+}}+\sqrt{\frac{\mu^{2} r_{+}^{2}}{16 r_{+}^{2}}+\frac{\mu^{2}}{2}}
$$$$
=\mu>\omega \text {. }
$$

Case II: $\omega<q Q / r_{+}<\mu$

We can also easily get

$$
\begin{aligned}
\omega_{+} & =\frac{q Q}{4 M}+\sqrt{\frac{q^{2} Q^{2}}{16 M^{2}}+\frac{\mu^{2}}{2}}>\frac{q Q}{4 r_{+}}+\sqrt{\frac{q^{2} Q^{2}}{16 r_{+}^{2}}+\frac{q^{2} Q^{2}}{2 r_{+}^{2}}} \\
& =q Q / r_{+}>\omega .
\end{aligned}
$$

So when $\omega$ satisfies the superradiance and the bound state conditions, $f(\omega)>0$. It implies that

$V^{\prime}(r \rightarrow \infty) \rightarrow 0^{-}$

This means there is no potential well when $r \rightarrow+\infty$. In the following, we will show that there is only one maximum outside the event horizon for $V$, no trapping potential exists which is separated from the horizon by a potential barrier and all non-extremal RN black holes are superradiantly stable.

The explicit expression of the derivative of the effective potential is

$$
\begin{aligned}
V^{\prime}= & -\frac{1}{\Delta^{3}}\left[\left(-4 M \omega^{2}+2 q Q \omega+2 M \mu^{2}\right) r^{4}\right. \\
& +\left[4 Q^{2} \omega^{2}+4 M Q q \omega-4 M^{2} \mu^{2}\right. \\
& \left.-2 Q^{2}\left(q^{2}+\mu^{2}\right)+2 l(l+1)\right] r^{3} \\
& +\left[-6 Q^{3} q \omega+6 M Q^{2} \mu^{2}-6 M l(l+1)\right] r^{2} \\
& +\left[2 Q^{4} q^{2}-2 Q^{4} \mu^{2}-4 M^{2}+4 Q^{2}\right. \\
& \left.+2\left(2 M^{2}+Q^{2}\right) l(l+1)\right] r+4 M^{3} \\
& \left.-4 M Q^{2}-2 M Q^{2} l(l+1)\right] .
\end{aligned}
$$

Defining a new variable $z=r-r_{-}$is convenient for us to study the property of the effective potential. Then Eq. (24) can be written as

$$
V^{\prime}(z)=\frac{-1}{\Delta^{3}}\left(a z^{4}+b z^{3}+c z^{2}+d z+e\right),
$$

where

$$
\begin{aligned}
a= & -4 M \omega^{2}+2 q Q \omega+2 M \mu^{2}, \\
c= & 12 r_{-}\left\{-4 r_{-}^{2} \omega^{2}+6 r_{-} \omega q Q\right. \\
& \left.-\left[2 q^{2} Q^{2}+\left(3 r_{+} r_{-}+r_{-}^{2}\right) \mu^{2}\right]\right\}-3\left(r_{+}-r_{-}\right) l(l+1),
\end{aligned}
$$

$$
e=2 r_{-}^{2}\left(r_{+}-r_{-}\right)\left(\omega r_{-}-q Q\right)^{2}+\frac{1}{2}\left(r_{+}-r_{-}\right)^{3}
$$

From the asymptotic behaviors of the effective potential at the inner and outer horizons and infinity, we know that there are at least two roots for $V^{\prime}(z)=0$ when $z>0$. If a trapping potential existed, there would be at least four positive roots for $V^{\prime}(z)=0$. Next, we will demonstrate that it is impossible for the equation $V^{\prime}(z)=0$ to have four positive roots when the superradiance condition (13) and the bound state condition (12) are satisfied.

Because we are just concerned with the roots of $V^{\prime}(z)=$ 0 , the numerator of Eq. (25) will be considered only. We denote the roots of $V^{\prime}(z)=0$ by $\left\{z_{1}, z_{2}, z_{3}, z_{4}\right\}$ and $z_{1}, z_{2}$ are the two known positive roots $\left(r_{-}<z_{1}<r_{+}, r_{+}<z_{2}<\right.$ $+\infty)$. According to the Vieta theorem, we have the following relations for the roots:

$$
\begin{aligned}
z_{1} z_{2}+z_{1} z_{3}+z_{1} z_{4}+z_{2} z_{3}+z_{2} z_{4}+z_{3} z_{4} & =\frac{c}{a} \\
z_{1} z_{2} z_{3} z_{4} & =\frac{e}{a} .
\end{aligned}
$$

The coefficient $a(=f(\omega))$ has been proved to be positive before. Because $r_{+}>r_{-}$, it is also easy to see that

$e>0$.

So from Eq. (30), we find that if $z_{3}, z_{4}$ are two real roots, they must be both positive or both negative.

Taking the superradiance and bound state conditions into account, we will use an algebraic method to prove $c<0$ for the full parameter space of the charged massive scalar perturbation and non-extremal RN black holes. So $z_{3}, z_{4}$ cannot be both positive, there is no trapping well outside the horizon and the RN black hole is superradiantly stable. This is the main result of this paper.

The final term of $c$ is non-positive, so in order to prove $c<0$, we just need to prove

$$
\begin{aligned}
& 12 r_{-}\left\{-4 r_{-}^{2} \omega^{2}+6 r_{-} \omega q Q-\left[2 q^{2} Q^{2}+\left(3 r_{+} r_{-}+r_{-}^{2}\right) \mu^{2}\right]\right\} \\
& \quad<0
\end{aligned}
$$

i.e.

$$
\begin{aligned}
g(\omega) & =-4 r_{-}^{2} \omega^{2}+6 q Q r_{-} \omega-\left[2 q^{2} Q^{2}+\left(3 r_{+} r_{-}+r_{-}^{2}\right) \mu^{2}\right] \\
& <0 .
\end{aligned}
$$


Regarding $g(\omega)$ as a quadratic function of $\omega$, when the discriminant of $g(\omega)$ (denoting it by $\Delta^{\prime}$ )

$\Delta^{\prime}=4 r_{-}^{2}\left[q^{2} Q^{2}-4\left(3 r_{+} r_{-}+r_{-}^{2}\right) \mu^{2}\right]<0$,

we have $g(\omega)<0$ because the coefficient of $\omega^{2}$ is negative.

Below we discuss the case $\Delta^{\prime} \geq 0$. According to the properties of a quadratic function, when $\Delta^{\prime} \geq 0$ is satisfied, there are two positive roots of $g(\omega)$, which are denoted by $\omega_{1}$ and $\omega_{2}$, respectively, and $\omega_{1} \leq \omega_{2}$. To demonstrate $g(\omega)<$ 0 , we only need to demonstrate $0<\omega<\omega_{1}$ when the superradiance and bound state conditions are satisfied. We do this for two possible cases. It is easy to get

$\omega_{1}=\frac{3 q Q-\sqrt{q^{2} Q^{2}-4\left(3 r_{+} r_{-}+r_{-}^{2}\right) \mu^{2}}}{4 r_{-}}$.

According to $\Delta^{\prime} \geq 0$ and $r_{+}>r_{-}$, we can obtain

$q Q>4 \mu r_{-}$.

Case I: $\omega<\mu \leq q Q / r_{+}$,

$$
\begin{aligned}
\omega_{1} & =\frac{3 q Q-\sqrt{q^{2} Q^{2}-4\left(3 r_{+} r_{-}+r_{-}^{2}\right) \mu^{2}}}{4 r_{-}} \\
& >\frac{q Q}{2 r_{-}}>2 \mu>\mu>\omega .
\end{aligned}
$$

Case II: $\omega<q Q / r_{+}<\mu$,

It is also easy to get

$\mu^{2} r_{+}^{2}>q^{2} Q^{2}>16 \mu^{2} r_{-}^{2}$,

so that

$r_{+}>4 r_{-}$.

Then we have

$$
\begin{aligned}
\omega_{1} & =\frac{3 q Q-\sqrt{q^{2} Q^{2}-4\left(3 r_{+} r_{-}+r_{-}^{2}\right) \mu^{2}}}{4 r_{-}} \\
& >\frac{q Q}{2 r_{-}}>\frac{q Q}{4 r_{-}}>\frac{q Q}{r_{+}}>\omega .
\end{aligned}
$$

The proof is thus completed.

In summary, we study the superradiant stability of nonextremal charged RN black holes against a charged massive scalar perturbation. Using an algebraic method, we demonstrate analytically that when the superradiance condition (13) and the bound state condition (12) are satisfied by the scalar perturbation and black holes, there is no trapping well out- side the event horizon, that is separated from the horizon by a potential barrier. So we conclude that all the nonextremal charged RN black holes are superradiantly stable against charged massive scalar perturbations.

Acknowledgments This work is supported partially by the Natural Science Foundation of Guangdong Province (No. 2016A030313444).

Open Access This article is distributed under the terms of the Creative Commons Attribution 4.0 International License (http://creativecomm ons.org/licenses/by/4.0/), which permits unrestricted use, distribution, and reproduction in any medium, provided you give appropriate credit to the original author(s) and the source, provide a link to the Creative Commons license, and indicate if changes were made.

Funded by SCOAP 3 .

\section{References}

1. T. Regge, J.A. Wheeler, Phys. Rev. 108, 1063 (1957)

2. C.A. Manogue, Ann. Phys. 181, 261 (1988)

3. W. Greiner, B. Mller, J. Rafelski, Quantum Electrodynamics of Strong Fields (Springer-Verlag, Berlin, 1985)

4. V. Cardoso, O.J.C. Dias, J.P.S. Lemos, S. Yoshida, Phys. Rev. D 70, 044039 (2004)

5. V. Cardoso, O.J.C. Dias, J.P.S. Lemos, S. Yoshida, Phys. Rev. D 70, 049903 (2004, Erratum)

6. R. Penrose, Revista Del Nuovo Cimento 1, 252 (1969)

7. D. Christodoulou, Phys. Rev. Lett. 25, 1596 (1970)

8. C.W. Misner, Phys. Rev. Lett. 28, 994 (1972)

9. Y.B. Zeldovich, Pisma Zh. Eksp. Teor. Fiz. 14, 270 (1971) [JETP Lett. 14, 180 (1971)]

10. Y.B. Zeldovich, Zh. Eksp. Teor. Fiz. 62, 2076 (1972) [Sov. Phys. JETP 35, 1085 (1972)]

11. J.M. Bardeen, W.H. Press, S.A. Teukolsky, Astrophys. J. 178, 347 (1972)

12. J.D. Bekenstein, Phys. Rev. D 7, 949 (1973)

13. W.H. Press, S.A. Teukolsky, Nature (London) 238, 211 (1972)

14. H. Furuhashi, Y. Nambu, Prog. Theor. Phys. 112, 983 (2004)

15. S.R. Dolan, Phys. Rev. D 76, 084001 (2007)

16. S.R. Dolan, Phys. Rev. D 87, 124026 (2013)

17. V. Cardoso, O.J.C. Dias, Phys. Rev. D 70, 084011 (2004)

18. V. Cardoso, S. Yoshida, JHEP 0507, 009 (2005)

19. V. Cardoso, O.J.C. Dias, S. Yoshida, Phys. Rev. D 74, 044008 (2006)

20. R. Li, Phys. Lett. B 714, 337 (2012)

21. R. Li, Eur. Phys. J. C 73, 2274 (2013)

22. S.-J. Zhang, Q. Pan, B. Wang, E. Abdalla, J. High Energy Phys. 09, 101 (2013)

23. J.G. Rosa, J. High Energy Phys. 02, 014 (2013)

24. S. Hod, O. Hod, Phys. Rev. D 81, 061502 (2010)

25. J.C. Degollado, C.A.R. Herdeiro, Gen. Relat. Gravity 45, 2483 (2013)

26. C.A.R. Herdeiro, J.C. Degollado, H.F. Rnarsson, Phys. Rev. D 88, 063003 (2013)

27. S. Hod, Phys. Lett. B 708, 320 (2012)

28. S. Hod, Phys. Lett. B 713, 505 (2012)

29. S. Hod, Phys. Lett. B 718, 1489 (2013)

30. R. Li, Phys. Rev. D 88, 127901 (2013)

31. S. Hod, Phys. Rev. D 91, 044047 (2015) 